\title{
Peer Network Processes in Adolescents' Health Lifestyles
}

\author{
jimi adams, \\ Department of Health \& Behavioral Sciences, University of Colorado Denver \\ Institute of Behavioral Science, University of Colorado Boulder \\ Elizabeth M. Lawrence \\ Department of Sociology, University of Nevada, Las Vegas \\ Joshua A. Goode, \\ Department of Sociology, University of Colorado Boulder \\ Institute of Behavioral Science, University of Colorado Boulder \\ David R. Schaefer \\ Department of Sociology, University of California, Irvine \\ Stefanie Mollborn \\ Department of Sociology, University of Colorado Boulder \\ Institute of Behavioral Science, University of Colorado Boulder
}

Accepted version, forthcoming in Journal of Health \& Social Behavior

Corresponding Author: jimi adams, Campus Box 188, PO Box 173364, Denver, CO 80217-3364

Email: jimi.adams@ucdenver.edu

Word Count: 9,674 (including title page, abstract, text, notes, references, tables, \& figures) 


\title{
Peer Network Processes in Adolescents' Health Lifestyles
}

\author{
ABSTRACT \\ Combining theories of health lifestyles — interrelated health behaviors arising from group-based \\ identities — with those of network and behavior change, we investigated network characteristics \\ of health lifestyles and the role of influence and selection processes underlying these \\ characteristics. We examined these questions within two high schools using longitudinal, \\ complete friendship network data from the National Longitudinal Study of Adolescent to Adult \\ Health. Latent class analyses characterized each school's predominant health lifestyles using \\ several health behavior domains. School-specific stochastic actor-based models evaluated the \\ bidirectional relationship between friendship networks and health lifestyles. Predominant \\ lifestyles remained stable within schools over time, even as individuals transitioned between \\ lifestyles. Friends displayed greater similarity in health lifestyles than non-friend dyads. \\ Similarities resulted primarily from teens' selection of friends with similar lifestyles, but also \\ from teens influencing their peers' lifestyles. This study demonstrates the salience of health \\ lifestyles for adolescent development and friendship networks.
}

\section{Keywords}

social networks, health lifestyles, adolescents, latent class analysis, stochastic actor-based models 
Health lifestyles are defined as clusters of health behaviors that co-occur in patterned ways, undergirded by group-based identities and norms (Cockerham 2005; Mollborn et al. 2014a). Health lifestyles offer a conceptual framework for understanding patterns of health behaviors, shedding light on persistent disparities in health behaviors, and explaining why such behaviors resist change. The health lifestyles that individuals adopt across the life course are influenced by social factors, with consequences for several important health outcomes (Burdette et al. 2017; Daw, Margolis, and Wright 2017; Lawrence, Mollborn, and Hummer 2017).

Health lifestyles theory emphasizes both the associations among various behaviors and the importance of social identities for the development of lifestyles (Cockerham 2005; Frohlich and Potvin 1999; Krueger, Bhaloo, and Rosenau 2009). Most notably for our purposes, Cockerham (2005) theorized the importance of peer effects among the social processes contributing to health lifestyle development, ranging from those based in social structure (e.g., socioeconomic status, demographics) to socialization and experiences (familial norms, formal education, etc.; Cockerham 2013). Peer effects represent an interesting blend of agency and structure because people both choose their peer groups and are influenced by them. However, despite early theoretical formulations anticipating peer effects in the construction, maintenance, and reproduction of health lifestyles, these effects have not been empirically tested.

We seek to assess whether health lifestyles display network effects consistent with theoretical expectations. "Network assortativity" describes a general pattern whereby friends exhibit elevated similarity on a range of attributes, including health behaviors like physical activity (de la Haye et al. 2011) and substance use (Haas and Schefer 2014; Kirke 2004); and core statuses that animate sociological research—gender (Kandel 1978), race (Moody 2001), and religious beliefs (adams, Schaefer, and Ettekal 2020). Beyond documenting the ubiquity of these 
patterns, network research can theoretically and empirically disentangle the causal processes responsible for observed assortativity (McPherson, Smith-Lovin, and Cook 2001). To identify these processes, models separate peer influence - people adopting the behaviors of their friends - from homophilous selection— choosing friends with similar behaviors (Steglich, Snijders, and Pearson 2010). Our primary research question therefore asks whether health lifestyles display network assortativity and if so, whether peer influence and homophilous selection processes underlie health lifestyle development.

We use data from two schools in the National Longitudinal Study of Adolescent to Adult Health (“Add Health”). We identify health lifestyles using latent class analysis (McCutcheon 1987; Vermut and Magidson 2002) that investigates combinations of ten health behaviors. The analyses identify meaningful clusters of behaviors that differ across schools but are consistent over time within each school, even when individuals change their own health lifestyles. Further, these health lifestyles display assortativity: health lifestyles are more similar among friends than other peers. We use stochastic actor-based models (Snijders 2011; Steglich et al. 2010) to assess peer influence and homophilous selection, finding that both processes contribute to observed assortativity among peers' health lifestyles. We decompose these results, finding that homophilous selection comprises the largest effect. We conclude that social networks and health lifestyles are intertwined.

\section{BACKGROUND}

\section{Health Lifestyles}

Health behaviors are not adopted in isolation. Adolescent health behaviors frequently cooccur (Brener and Collins 1998). Sometimes, co-occurring behaviors trend healthy or unhealthy. 
For example, adolescents who engage in risky sexual activity are also more likely to engage in substance use and fighting (Zweig, Phillips, and Lindberg 2002). Other times, co-occurring behaviors are a mix of healthy and unhealthy. For example, a large group of U.S. adolescents combine the most favorable exercise levels and dietary behaviors with the highest rate of binge drinking (Burdette et al. 2017). Research and prevention efforts can better explain adolescents' engagement in particular behaviors if they explicitly consider these complex interrelationships and their underlying influences and contexts.

Health lifestyles — interrelated health behaviors — are collective, reflecting group-based identities (Williams 1995). A particular clustering of behaviors reflects group membership (Sussman et al. 2007). For example, adolescents who consider themselves "jocks" and whose social circle consists of other "jocks" may engage in high levels of physical activity, unsafe habits, and binge drinking (Barber, Eccles and Stone 2001). In other words, discordant combinations of healthy and unhealthy behaviors may be explained by an underlying groupbased identity rather than by other characteristics such as personal commitment to maximizing healthy behavior (Stets and Burke 2000). Other group-based processes that shape behavior, such as norms, social learning, and role modeling, likely combine with—and are strengthened by— investment in a group-based identity. Together, these mechanisms of social influence can link group membership to a particular set of health behaviors.

\section{Health Lifestyles in Adolescence}

In adolescence, individuals initiate new health behaviors and increasingly make health behavior choices for themselves (Resnick et al. 1997). Multiple contexts are relevant for adolescent health behaviors, including families, neighborhoods, schools, and peers. Parents lay 
important foundations for the behaviors children adopt (Liefbroer and Elzinga 2012), dependent upon social, economic, and demographic factors (Mollborn et al. 2014c). In schools and neighborhoods, peers, adults, and structural features establish norms that influence behavior (Gest et al. 2011).

Therefore, peers likely influence health lifestyles, particularly among adolescents. Peers act as referents, role models, and information sources who reinforce or sanction behaviors (Stead et al. 2011; Valente 2010). Such peer influences can have long-lasting consequences, partly due to ways that health behaviors cluster together (Mollborn et al. 2014b) and mutually reinforce each other (Ennett et al. 2006). Lifestyle patterns across contexts have not previously been investigated empirically, but since the groups underlying health lifestyles differ across settings, we expect that health lifestyles will be specific to those settings. Because health lifestyle theory links lifestyles to group identities, we expect lifestyles to be structurally consistent across time within contexts (assuming group identities within that context remain stable). ${ }^{1}$ That is, even as individuals change their health behaviors over time, collective behavioral patterns should still reflect the lifestyle options available in that context. Therefore, the composition of lifestyles (i.e., structure) is recreated across time, even as the individuals adopting a given lifestyle change.

We therefore expect to find meaningful clusters of health behaviors, including some that are consistently healthy or unhealthy and others that are discordant. We also expect the compositions of health lifestyles to vary across contexts, but within a context, the composition of health lifestyles will be similar over time, even as individuals change their behaviors and lifestyles.

Peer Network Processes and Health 
Network research has consistently documented that people's social contacts include others who resemble themselves on a wide range of characteristics. This pattern is labeled "network assortativity" and is observed for numerous health behaviors and conditions (Schaefer and adams 2017, Valente 2010), including substance use (de la Haye et al. 2013; Haas and Schaefer 2014, Kirke 2004), physical activity (de la Haye et al. 2011), and psychological states such as depression (Schaefer, Kornienko, and Fox 2011). However, the vast majority of this research is conducted for single behaviors or conditions, with only one study examining multiple religious measures (adams et al. 2020). Thus, we first seek to identify whether health lifestyles display observed assortativity, as prior research has done for single behaviors.

Observed assortativity could result from several processes (Kandel 1978). Some assortativity is expected by chance alone due to the unequal distribution of characteristics in a population (Blau 1977; Marsden 1988), but two primary processes operate above and beyond this baseline: peer influence and homophilous selection.

Assortativity arises from peer influence when individuals adopt the behaviors or attitudes of their friends (Snijders 2011; Steglich et al. 2010). Peer influence is supported by sociological and psychological theories, including social learning from one's peers, normative influence, behavioral modeling, a need for belonging, and others (Brechwald and Prinstein 2011; Friedkin and Cook 1990). Peer influence assumes that if person $i$ names person $j$ as a friend at time 1, but they differ on a (changeable) attribute, $i$ will have an increased probability of changing their behavior to match $j$ 's at time 2 (see Column I of Figure 1). While theoretically appealing and frequently evoked to explain network similarity (Smith and Christakis 2008), influence is notoriously hard to identify empirically (Cohen-Cole and Fletcher 2008) and difficult to disentangle from homophilous selection (Shalizi and Thomas 2011). 


\section{Figure 1}

In contrast to peer influence, homophilous selection produces network assortativity when people are more likely to form new relationships with peers with whom they share characteristics (Kandel 1978). While network scholarship typically describes homophilous selection as a singular process, it is composed of two phenomena: the creation and dissolution of friendships (McPherson et al., 2001). The creation of friendships is depicted in Figure 1 (top row of homophilous selection). According to homophilous selection, if person $k$ and person $l$ share an attribute (e.g., a health lifestyle) but are not friends at time 1, there is an increased likelihood of $k$ and $l$ becoming friends by time 2 . Dissolution (bottom row of homophilous selection) represents the converse of this process. If persons $m$ and $n$ are friends at time 1 but differ on an attribute like health lifestyles, homophilous selection would expect a higher likelihood of their friendship dissolving (for further elaboration, see Snijders 2011).

\section{Summary}

Our overarching question is whether health lifestyles serve as a basis for homophilous selection and peer influence processes within adolescent social networks. To answer this question, we assessed whether health behaviors clustered together in empirically identifiable profiles of behaviors (i.e., "health lifestyles") within schools, evaluated if health lifestyles were more similar among friends than other peers (i.e,. assortativity on health lifestyles), and examined the extent to which peer influence and homophilous selection processes contributed to this similarity.

\section{DATA AND METHODS}


We used data from the two largest saturated schools in Add Health (Bearman et al. 1997). ${ }^{2}$ These schools (frequently referred to by the pseudonyms "Jefferson" and "Sunshine" High Schools) represent distinct social contexts and are common for benchmarking social network change estimation (Green et al. 2013; Haas and Schaefer 2014). Jefferson was a mostly white Midwestern school, and Sunshine was a much larger, ethnically diverse Western school. Our data came from two in-home interview surveys approximately one year apart: Waves I and II.

Our analyses proceeded in two primary steps which separately estimated health lifestyles, then modeled network dynamics using those health lifestyles. Given that we conceptualize health lifestyles as a context-specific phenomenon, we fit latent class analysis ("LCA") models using all adolescents with available data in each school, irrespective of their inclusion in subsequent models. ${ }^{3}$ The stochastic actor-based models ("SABM") included only those in the network data. The SABM sample was marginally younger at Wave I than the full sample, but otherwise comparable.

\section{Identifying Health Lifestyles with LCA}

We used latent class analysis (McCutcheon 1987; Vermut and Magidson 2002) to estimate health lifestyles from a comprehensive set of health behaviors. Following theoretical development and previous empirical work, we selected ten health indicators representing the range of behavioral domains previously identified as useful for defining adolescents' health lifestyles (Burdette et al. 2017; Cockerham 2005; Daw et al. 2017; Lawrence et al. 2017; Mollborn et al. 2014a). ${ }^{4}$

The first domain, substance abuse, was represented by four indicators. Smoking was coded as non-smoking, infrequent (1-11 days per month), and frequent smoking (12+ days). 
Drinking was coded as non-drinking (' 0 '), moderate (' 1 '), and heavy, binge, or problem drinking (' 2 ') in the past year. Heavy drinking was more than 8 drinks per week for women or more than 15 for men; binge drinking entailed drinking 5 or more drinks in a row; problem drinking identified social or physical problems resulting from alcohol consumption. Variables for other (chewing) tobacco and drug use were each coded as ' 1 ' if adolescents had used them within the past 30 days and ' 0 ' if they had not.

The second domain was activity level. One indicator summed how many times the respondent had engaged in different physical activities (bicycling, softball, etc.) during the past week, recoded into light (0-3), moderate (4-6), and heavy activity (7-15). The second indicator measured hours per week spent watching television or videos and playing video games, recoded into low ( 2 hours/day or less), moderate (greater than 2 and less than or equal to 4 hours), and high (greater than 4 hours).

The final four domains were represented by single indicators. Sexual activity measured the respondent's most recent sexual encounter with categories for no sex, sex with a condom, and sex without a condom. Safety captured respondents' seatbelt use, coded as '1' for always and ' 0 ' for others. The healthcare domain was represented by whether the respondent had received a checkup with a doctor and dentist within the past year, coded as ' 1 ' for those who had visited both and ' 0 ' for those who had not. Finally, sleep was coded as ' 1 ' for those who indicated that they received enough sleep and ' 0 ' for those who did not.

We fit separate LCA models for each school at each wave, using poLCA in R (Linzer and Lewis 2011), generating four sets of class assignments. LCA uses maximum likelihood to identify a categorical latent trait that underlies observed associations among indicators. The 
categorical latent variable is defined by the patterns of conditional probabilities among items, which are assumed to be conditionally independent given the categorical latent trait. In contrast to a regression approach which identifies individual associations with an outcome, LCA allows complex patterns to emerge from the data.

For each LCA model, we examined solutions for 2-12 classes and selected the best for use in network analysis based on substantive interpretation and fit statistics, including the Bayesian Information Criterion (BIC), Akaike Information Criterion (AIC), and $\mathrm{G}^{2}$ (Linzer and Lewis 2011; Vermut and Magidson 2002). ${ }^{5}$ We used the "classify-analyze" approach that separated the modeling into two steps (Bray, Lanza, and Xianming 2015), assigning individuals to the latent class with the largest posterior probability (i.e., "classify"), then estimating network models using this nominal variable (i.e., "analyze"). We employed this approach for two reasons. First, no modeling approach combines estimating latent classes with network dynamics into a single framework, allowing for estimating selection and influence in a manner akin to SABM. Second, our approach was a conservative test, as associations are expected to be biased downward (Bolck, Croon, and Hagenaars 2004). Average class assignment probabilities exceeded .80 (ranging from .81 to .86 ), suggesting minimal misclassification bias (Jung and Wickrama 2008).

\section{Modeling Network Dynamics with SABM}

We used these latent class assignments, or health lifestyles, to model friendship and health lifestyle co-evolution. The state-of-the-art approach to modeling such network dynamics is stochastic actor-based models (Snijders 2001, Steglich et al. 2010). SABM permits the simultaneous estimation of changes in friendships and class membership while allowing 
endogenous effects between the two. Thus, we tested how the evolving network structure affected health lifestyle class membership (peer influence), alongside the effects of health lifestyle on adolescents' choices of friends (homophilous selection).

The SABM aimed to recreate the network and class membership changes observed at Wave II. This was accomplished by specifying an objective function for each outcome (i.e., friendship network and lifestyle class). These two objective functions contained effects representing the mechanisms that drive change in network and lifestyle outcomes. The model assumes that individuals made changes to their outgoing network ties and behavior to maximize these objective functions. Estimation used an agent-based model to obtain parameter estimates, reflecting each effect's relative strength.

The network objective function estimated individuals' likelihood of forming or maintaining ties, relative to failing to form or dissolve ties respectively. We focused on whether friendships are more likely among individuals with the same health lifestyle class (homophilous selection). This process is illustrated in Figure 1, where adolescents $k$ and $l$ belonged to the same LCA class and became friends over time, whereas $m$ and $n$, who were members of different classes, dissolved their friendship. We represented this form of selection using the effect for same class membership, which measured whether the adolescents in each dyad were the same or different (Ripley et al. 2019). A positive parameter estimate for this effect indicates that dyads belonging to the same class were more likely to exhibit friendship over time (either by forming a tie or maintaining an existing tie).

The SABM behavior objective function predicts changes in individuals' behavior as a product of their own attributes, others' attributes (i.e., influence), and network structural features. SABMs have most often been used to model change in a single dichotomous or ordinal 
behavioral variable (Steglich et al. 2010); however, they can accommodate latent classes (adams et al., 2020), such as our categorical health lifestyles. To accomplish this, we adopted the strategy for modeling "behavioral" variables within an SABM as a two-mode network (Snijders, Lomi and Torló 2013), coding each adolescent as having a membership state for each of the classes (or categories of affiliation), with " 1 " coded for the assigned class and " 0 " coded for the other classes. There are two time points of these two-mode networks representing LCA class membership for each school. Peer influence was modeled using the "to" effect in the behavior function, while homophilous selection was modeled using the "from" effect in the network function (Ripley et al. 2019). The difference between this modeling strategy and the conceptualization of selection is represented in column II of Figure 1, where $i$ and $j$ are friends at T1 but have different lifestyle class memberships — where class is denoted as a membership (square), rather than a nodal attribute. We are primarily interested in whether adolescents adopted the same lifestyle class as their friends, expecting that adolescents chose the most common class among their friends. We tested this with an effect representing how many of each adolescents' current friends belonged to each class. A positive parameter estimate indicates that adolescents were more likely to choose a lifestyle class when they had more friends in the class. Given the evolving nature of the network and lifestyle classes, friends' lifestyles also changed endogenously.

The SABM assumes that changes in the network and behavior between the two observed waves of data occur in continuous time through a sequence of microsteps (adams and Schaefer 2018; Ripley et al. 2019) and that, during a given microstep, one actor can make one change to either the network or LCA class (i.e., change follows a Markov process). Rate functions corresponding to each network and behavior function indicate how many opportunities actors are 
given to make changes. We specified each rate function to be uniform across actors. We constrained the model such that adolescents could not have more than 10 outgoing ties (to be consistent with the Add Health data collection design) or 2 class memberships. This latter requirement was needed because the Markov assumption behind the SABM allows for only one change per microstep. Thus, individuals cannot simply switch from one class to another, as this would represent both the dissolution of a tie to one class and the creation of a tie to another class. By allowing up to 2 LCA-class memberships, adolescents in a given class can switch from their current class to another by either adding a tie to the new class (keeping their current membership until a later microstep) or dropping their current membership and adding the new membership in a later microstep. Negative outdegree effects serve as a deterrent to belonging to multiple classes.

Following SABM best practices, our models included additional controls. Standard individual-level controls were included in both the network and behavior functions for gender (female as reference category), age (in years at Wave I), race/ethnicity (for Sunshine only; nonHispanic white as reference), GPA (4-scale), and parents' highest level of completed education. We allowed each of these attributes to have a separate effect on each class. To specify this in the SABM, we set the healthy class as the reference and included interactions between dummy variables representing each other class and sociodemographic measure. Hence, main effects of attributes represent the effects of demographic (and other) variables on the likelihood of selecting the healthy class, while the interactions represent how the effect of the attribute on the likelihood of selecting the respective class departs from its effect on selecting the healthy class. As noted above, the behavior function includes an actor outdegree effect that represents the likelihood of observing any tie in the two-mode network. To allow class membership rates to 
differ, we treated the healthy class as the reference category and added interactions between dummy variables for the other classes and actor outdegree.

The network function included controls that captured the possibility that the attribute affected individuals' likelihood of being selected as a friend (i.e., attribute-based popularity), which, for parsimony, were dropped from the model if not significant (Ripley et al 2019). The network function also included controls for other normative friendship processes including: homophilous selection on other attributes (race/ethnicity, gender, academic achievement, and socioeconomic status), and endogenous network effects (e.g., reciprocity, which is the increased likelihood of $i$ nominating $j$ as a friend given $j$ 's nomination of $i$ ). The network function also included controls for structural opportunities for friendship with effects representing, for each dyad, whether or not they were in the same extracurricular activity (Schaefer et al., 2011) or were coursemates (weighted; Frank et al. 2008).

Additional controls addressed alternative mechanisms. For instance, friendships developed in contexts like extracurricular activities or classrooms probably exhibit assortativity on attributes associated with membership in the context (e.g., friends are likely to be similar in physical activity when their friendship developed within a sport context). Assortativity can also arise when a friendship emerges between two individuals because of a common friend (i.e., transitivity, the increased likelihood of a friendship between pairs of people who share other friends in common). Transitivity amplifies levels of assortativity if the common friend shares a common attribute with the two friends, in which case the process of transitivity can create network assortativity in the absence of homophilous selection (Moody 2001; Wimmer and Lewis 2010). Indegree-popularity accounts for one's general tendency to receive tie nominations given how many ties one has already received, outdegree-popularity represents one's tendency to send 
ties given how many ties one has received, and indegree-activity is one's general tendency to send ties given how many ties one has received (Ripley et al. 2019). By specifying these effects, our SABM was able to differentiate which among the various selection mechanisms was responsible for the observed network patterns.

We used a decomposition approach (Steglich et al. 2010) to ascertain the relative importance of peer influence versus homophilous selection in producing observed health lifestyle assortativity. This multi-step procedure used the agent-based model core of the SABM to simulate network-lifestyle co-evolution based on estimated model parameters. We compared the simulated levels of assortativity for the full estimated model with levels observed in a series of simulations that systematically constrained individual key effects to zero (i.e., peer influence, homophilous selection). The magnitude of the decrease in assortativity when an effect was constrained to zero indicates the mechanism's relative contribution to observed assortativity. This procedure is repeated for each set of processes labeled in Figure 4. We measured lifestyle assortativity using $\alpha$, as described earlier, and averaged results across 1,000 simulations per specification for each school.

We estimated the SABMs separately by school given that they represent independent contexts with no possibility of ties between schools. High school seniors at Wave I were excluded from analysis since they were not observed at Wave II. We estimated SABMs with RSiena (version 1.1-232, Ripley et al. 2019), using standard RSiena settings to impute data for anyone else missing from Wave II (i.e., those lost through attrition) and for other forms of missing data (Huisman and Steglich 2008). We conducted post-estimation checks to ensure adequate model convergence and goodness of fit (Lospino 2012); see Appendix Figures A1 and A2. 


\section{FINDINGS}

Table 1 provides descriptive statistics for students in Jefferson and Sunshine High Schools, by wave. Because Sunshine only included grades 10-12, its attendees were older than Jefferson's. As previously shown (Moody 2001), these schools' race/ethnic composition differed substantially. The prevalence of several behaviors was similar across schools, while others differed. For example, similar proportions of students in each school reported engaging in no sexual activity and condom use at last sex among the sexually active. In contrast, Jefferson students had lower average screen time but higher rates of substance use (smoking, drinking, and other tobacco use) than Sunshine students.

\section{Table 1}

\section{Health Lifestyles}

We estimated one latent class model for each school for each wave. For each model, we selected a three-class solution as the best fitting. Three of the four models (Jefferson at both Waves I and II, Sunshine at Wave I), the adjusted BIC (Supplementary Table A1) indicated that a three-class solution best fit the data. Moreover, within each solution, the classes had interpretable distributions of variables across the indicated classes. For Sunshine at Wave II, the adjusted BIC suggested that the best-fitting solution had two classes, but we chose the three-class solution because it showed a trivial statistical fit difference compared to the two-class solution, it was compositionally comparable to the three-class solution for the other models, and a consistent solution set allowed us to more parsimoniously address our aims of examining network selection and influence processes. ${ }^{6}$ 
Figure 2 displays results from the three-class solutions for each school by wave. These radar plots correspond to class-conditional response probabilities (rho), which can be interpreted as the probability of class members being in the top-coded category for each health behavior (adams and Lippert 2019). The plots present items normalized within a 0-1 range and standardized in a consistent (healthy) direction. ${ }^{7}$ The Appendix describes the standardization procedure and provides the full set of class-conditional response probabilities (Appendix Table A2).

Figure 2

Figure 2 conveys several key points. First, the health lifestyles reflect concordant and discordant groups that differ somewhat by school. We summarize the three classes identified in each school at both waves as mostly healthy, mostly unhealthy, and discordant. While we rely on these shorthand labels, patterns are more complex. Members of the healthy class typically exhibited some unhealthy behaviors, those in the unhealthy classes reported some healthier behaviors, and the discordant groups reflected combinations of healthy and unhealthy patterns. For example, members of the Wave I Jefferson discordant class were substantially more likely to use chewing tobacco than the unhealthy class. ${ }^{8}$ Consistent with our expectations, the complex patterns of behaviors within each latent class provide empirical support for the health lifestyles approach within these two schools (e.g., as opposed to an index).

In each model, the healthy group had high rates of nonsmokers, nondrinkers, those who have not had sex, and those who always wore a seatbelt. The unhealthy groups all showed high rates of drug/alcohol/smoking use, not using a condom during last sex, and lower levels of physical activity. However, the patterns in the discordant groups diverged between the two schools. Those in the discordant group at Jefferson showed high rates of physical activity, 
getting enough sleep, and visiting the doctor and dentist, but also substantial substance use, including smoking, drinking, tobacco chewing, and drug use. In contrast, those in the discordant group at Sunshine had fairly low rates of substance use, but also low rates of physical activity, getting enough sleep, and use of health care. In sum, those in the discordant class at Jefferson were slightly more likely to actively engage in behaviors (whether healthy or not), while those in the discordant class at Sunshine were more passive (avoiding behaviors, whether healthy or unhealthy). These divergent patterns suggest that prevalent adolescent health lifestyles varied by school context. ${ }^{9}$

Health lifestyle patterns over time differed by school. In Jefferson, the prevalence of each health lifestyle remained similar over time, with a slight decrease in the percentage in the healthy class ( $49 \%$ to $41 \%$ ) corresponding to an increase in the unhealthy class (33\% to $40 \%$ ), while the discordant class remained stable (19\%). Sunshine displayed greater shifts in prevalence, with the healthy group dropping from $53 \%$ to $26 \%$, corresponding with increases in the mixed class (15\% to $35 \%$ ), and a slight increase in the unhealthy class (32\% to 39\%). Surprisingly, even more students changed their health lifestyles than these compositional shifts indicated: About $47 \%$ of Jefferson students and $66 \%$ of students in Sunshine changed their health lifestyles from Wave I to Wave II.

Although many students changed health lifestyle, the school-level prevalence of each health behavior displayed remarkable sample-level stability across waves. For example, rates of getting enough sleep were 69\% in Wave I and 71\% in Wave II for Jefferson, and 64\% and 62\% in Sunshine. The similar prevalence in Waves I and II obscures the changes: 25\% of Jefferson and 29\% Sunshine students changed their sleep status (either from enough to not enough or from 
not enough to enough) between the waves. These behavioral and lifestyle changes are consistent with prior literature (Daw et al. 2017). ${ }^{10}$

Importantly, the contextual composition of classes remained relatively robust to these underlying individual changes. That is, the composition of health lifestyle classes appears to have been consistent over time within contexts-with the noted exception of Sunshine's discordant class. This class remained "passive" by not engaging in behaviors that were either health promoting or compromising but became more passive across waves (e.g., decreasing in both drinking and physical activity). Since we separately fit models for each school at each wave, this is a novel, empirically observed contextual consistency in class compositions, not an artifact of modeling.

\section{Networks}

Table 2 describes the schools' friendship networks, both of which exhibited distinct network characteristics. The out-degree values indicate the proportion of friends in a school, and the lower values for Sunshine demonstrate that Sunshine students reported substantially fewer within-school friends than their Jefferson counterparts. ${ }^{11}$ Consistent with prior research, Jefferson students had a higher proportion of their friends within the school, while Sunshine provided a more porous boundary for adolescents' reported friendships (Moody 2001). The Jaccard indices (.34 for Jefferson, .21 for Sunshine) indicate that each school had sufficient stability in the observed friendship networks to justify our SABM approach. ${ }^{12}$

\section{Table 2}

Table 2 also provides evidence that health lifestyles displayed strong network assortativity in each school: Friends were more likely to share a health lifestyle. The LCA-Class 
assortativity, or $\alpha$ segregation index, measures observed health lifestyle assortativity in each school (Moody 2001). ${ }^{13}$ The odds ratios indicate that in each school, at both waves, the odds of a friendship between adolescents in the same LCA class were significantly greater than adolescents with different classes. For example, at Wave I, the odds of a same-class tie were $85 \%$ greater in Jefferson and $62 \%$ more likely in Sunshine compared to ties between adolescents from different classes. We used the SABMs to explain this strong health lifestyle assortativity.

\section{Network Processes for Health Lifestyles}

We estimated separate SABMs for each school to test homophilous selection and influence processes, while controlling for standard potentially confounding factors. For brevity, we focus our discussion on the health lifestyle-related effects shown in Figure 3 that correspond to our focal questions. While we present these estimates in a single figure, we caution readers to not directly compare effect sizes in Figure 3, as the scales and control terms are specific to each model and not comparable. Full model specifications are available in Appendix Table A3; controls operated consistently with prior studies.

In the behavior function, our key process of interest was peer influence, represented by the "Friend with same LCA Class" effect. In both schools, this effect was statistically significant (i.e., different from zero, $\mathrm{p}<0.001)$ and positive, indicating that adolescents were more likely to adopt a lifestyle class as their number of friends in that class increased. In Jefferson, the influence estimate of .429 indicates that for each additional friend an adolescent had in a particular lifestyle class, the odds of choosing that class increased by 53\% (exp[.429]), all else being equal. For Sunshine, the estimate reflects a 30\% increase (exp[.26]) in selecting a class for 
each additional friend the adolescent had with that class. These effects offer evidence of peer influence on health lifestyles in both schools.

\section{Figure 3}

Turning to the network function, homophilous selection is represented by the "LCA (same category)" effect. In both schools, this effect was positive and statistically significant. These coefficients can be interpreted as the difference in the likelihood of a friendship being formed or maintained for two adolescents with the same lifestyle class (coded 1) vs. two adolescents from different classes (coded 0). Thus, for Jefferson, the odds of a tie between adolescents with the same class were 2.7 (exp[1.008]) times the odds of a tie between dissimilar adolescents, all else being equal. Sunshine, while demonstrating a large and significant selection effect, also showed a wide confidence interval around this estimate.

To compare across models and parameters, our final step decomposed the relative contributions of homophilous selection and peer influence to health lifestyle assortativity; see Figure 4. In both schools, homophilous selection carried the greatest weight in producing lifestyle assortativity, responsible for $68 \%$ and $55 \%$ of assortativity in Sunshine and Jefferson, respectively. In contrast, peer influence was only responsible for $11 \%$ and $20 \%$ of assortativity, respectively. The proportions for trend indicate that $5.5 \%$ and $10 \%$ of assortativity change was due simply to changes in network and individual class memberships. Controls accounted for only $2-3 \%$ of changes in lifestyle assortativity. Finally, 12-13\% of assortativity could not be precisely allocated to any of these (Steglich et al. 2010). In sum, the observed assortativity on health lifestyles in Jefferson and Sunshine high schools appears to have been primarily the result of homophilous selection though also exhibiting significant peer influence. These results offer 
strong and consistent support for our approach in identifying network processes underlying health lifestyle-based behavioral clusters.

\section{Figure 4}

\section{DISCUSSION}

Our aim was to understand if and how network processes are related to adolescent health lifestyle development. We found that adolescent health lifestyles were specific to context but structurally similar over time, as the lifestyle compositions differed across the two schools but were mostly consistent within schools over time. These health lifestyles displayed strong network assortativity, which was the result of both peer influence and homophilous selection processes, while the latter was stronger. We connect these results to prior research and draw out their implications below.

\section{Health Lifestyles}

Our LCA-identified health lifestyles showed similar patterns to previous research using Add Health data (Burdette et al. 2017; Daw et al. 2017; Lawrence et al. 2017). The nature and composition of our discordant classes were somewhat similar to Burdette et al.'s (2017) larger Add Health sample. Teen respondents' relatively high rates of change in their health lifestyles from one year to the next is also consistent with trajectories of lifestyle development over the life course (Daw et al. 2017; Frech 2012). Our study is the first to model health lifestyle classes within local settings. We theorized that lifestyles would vary across schools, and our results bear out this conclusion. Further research across a broader range of contexts will likely shed light on contextual determinants of health lifestyles. Our study is also the first to model health lifestyles 
within settings longitudinally, showing that localized health lifestyles remain largely consistent over time even as individuals flow in and out of these lifestyles. This finding suggests that health lifestyles reflect a range of normative options and bolsters theoretical foundations for the structural significance of health lifestyles.

\section{Friendship Network Processes and Health Lifestyles}

This study demonstrates strong evidence that network processes are an important facet of adolescent health lifestyle development. Our results show strong observed network assortativity, which is the result of both peer influence and homophilous selection processes. While previous theorization of health lifestyles articulated the likely role of peer processes in their development and maintenance (Cockerham 2005), these processes had not been previously empirically tested. Peer influence has been shown to be influential for individual health behaviors in adolescence (Stead et al. 2011). Documenting peer influence on health lifestyles supports the group-level nature of health lifestyle theorization - with peer group members influencing one another's health lifestyles —-whereas previous research has tended towards individual-level measurement. Homophilous selection, whereby adolescents form or dissolve friendship ties based on health lifestyles, was particularly strong. This finding is in line with previous research demonstrating that health lifestyles are a form of status signaling and distinction that shape social ties and social capital (Mollborn, Rigles, and Pace 2020). Homophilous selection also highlights the importance of agency for health lifestyle formation, as adolescents selected friendships on the basis of others' health lifestyles.

This study contributes to an increasing consideration of whether homophilous selection (Lewis and Kaufman 2018) and/or peer influence (Goldberg and Stein 2018) may operate over 
other clusters of behaviors or attitudes, rather than for single behaviors at a time (Strang and Soule 1998). Links between networks and single behaviors have longstanding theoretical and empirical bases, and shifting the paradigm towards clusters will not be easy. However, our results suggest such a shift can produce meaningful and useful results. As one of the first investigations of network selection and influence on clusters of behaviors (see also adams et al, 2020), our approach complements existing strategies focused on individual behaviors. However, many mechanisms underpinning social influence may work equally well for clusters of behaviors as for individual behaviors.

Focusing on the link between networks and single characteristics simplifies methodological strategies (e.g., it is easier to collect data that focus on a single domain at a time, statistical frameworks are more parsimonious, etc.). Modeling clusters of behaviors, while theoretically supported, may not be methodologically expedient for many researchers. An important step will be to develop tests to compare network characteristics and effects for individual characteristics versus clusters of behaviors as competing models. In such efforts, researchers should not assume that all clusters of behaviors will exhibit similar network effects to what we observe for health lifestyles. Health lifestyles have a robust theoretical and empirical underpinning for their composition and construction. Therefore, researchers should focus on clusters with similarly firm conceptual foundations, rather than indiscriminately looking for network effects on other clusters.

\section{Limitations and Future Research}

As the first study to identify network processes for health lifestyles, these analyses raise questions that are unanswerable with our data, providing future opportunities for health lifestyles and network research. Unfortunately, these analyses' need for a combination of health and 
complete network data over multiple waves sets a high bar for data requirements, which few studies achieve. Rather than lamenting data limitations, we hope our work spurs a continued expansion of data of this sort, spanning different temporal and spatial contexts (e.g., Franken et al. 2016, Moody et al. 2010). We found both similarities and differences in the health lifestyles and network effects in two schools with vast racial/ethnic, socioeconomic, and geographic differences, bolstering our expectations that future extensions will find informative and interesting patterns. We encourage researchers to further document and explain how and why lifestyles differ across contexts.

We encourage future research to explore heterogeneity in dynamic network processes, including whether the processes differ across lifestyle types. For example, the composition of classes differed by gender in Sunshine, with more girls in the discordant class. Future work could seek to identify whether such differences are driven by network processes. It may also be that different compositions across contexts (e.g., varied distributions of the same health lifestyles) may differentially alter the class-specific nature of peer influence and homophilous selection effects (adams and Schaefer 2016; McFarland et al. 2014; Mollborn et al. 2020).

\section{CONCLUSION}

Our study demonstrates that health lifestyles and adolescent peer network processes are deeply intertwined, with each shaping the other to some extent. This study combined group-based clusters of behaviors with network processes to understand how health lifestyles, a meaningful and important concept in social research, develop in specific settings. Our results demonstrate the salience of health lifestyles for adolescent development and friendship networks, with important implications for conceptualizing and modeling peer network processes more broadly. 
An important consideration is whether the attributes that are diffusing and/or selected upon are individual (behaviors, attitudes, etc.) versus collective in nature (e.g., lifestyles). We encourage future consideration of how contextual features or other population characteristics (e.g., sociodemographic composition) may moderate how such peer network processes play out across local settings. Bringing together these theoretically and methodologically advanced ideas will further our understanding of how and why individuals adopt different behaviors. 


\section{NOTES}

1. We conceptualize local contexts as schools, as these are primary normative and behavioral environments for adolescents. Alternative conceptualizations (and modeling approaches) may be appropriate for other populations.

2. "Saturated" indicates that the survey was targeted to the complete student body, providing information on complete peer networks. The saturated design was used for more than 100 schools at the wave I in-school survey, and 16 schools for two additional in-home waves. The two schools we studied were larger and had more complete network data than the other 14 schools with longitudinal network data.

3. Alternate specifications estimated LCA restricted to the analytic sample used in the SABMs, with substantively similar results. We chose to estimate LCA for the full sample given the collective nature of health lifestyles.

4. Despite identifying these indicators within behavioral domains, model estimation does not assume statistical dependency between items within the same domain.

5. We present the fit statistics for the first eight of these in Appendix Table A1.

6. We also fit LCA models enforcing consistency across the waves (i.e., collapsing estimation across waves), as a robustness check. This approach resulted in similar LCA classes for Jefferson, while Sunshine exhibited a 4-class best fitting solution. SABM results using these alternate LCA specifications for modeling the focal homophilous selection and peer influence effects were substantively similar to those presented.

7. This normalization and standardization are for presentation purposes only. LCA models were fit on untransformed behavioral indicators. 
8. By Wave II, this difference remained in the same direction, but was diminished in magnitude.

9. These findings are suggestive of potentially important contextual differences in health lifestyle composition. However, more systematically comparing contextual compositional differences requires a larger sample of saturated schools than is available in Add Health $(\mathrm{N}=16)$.

10. We also performed ancillary analyses to examine whether LCA class assignment, or changes, hinged on a select set of behavioral indicators. These analyses showed that attempting to "explain" the changes observed in LCA class assignment—and transitions between classesdepended on multiple behavioral differences/changes, and was not attributable to single (or even a small subset of) behaviors. These results further support our focus on health lifestyles, rather than on individual behaviors.

11. While adolescents had the opportunity to nominate friends from outside the school, those nominations are excluded from our analysis since we have no information on their attributes.

12. The general rule of thumb is a Jaccard index greater than .2 is necessary for proper estimation (Snijders et al. 2010).

13. This index provides the properly conditioned form for asking how assortative ties are, given the general tendency for ties to form and the distribution of the attribute in the network (Bojanowski and Corten 2014). 


\section{REFERENCES}

adams, jimi and Adam M. Lippert. 2019. "Under the Radar: Simplifying the Representation of Latent Class Characteristics.” Socius (5):1-3.

adams, jimi and David R. Schaefer. 2016. "How Initial Prevalence Moderates Network-Based Smoking Change: Estimating Contextual Effects with Stochastic Actor Based Models." Journal of Health and Social Behavior 57(1):22-36.

adams, jimi, and David R. Schaefer. 2018. "Visualizing Stochastic Actor-Based Model Microsteps." Socius (4):1-3.

adams, jimi, David R. Schaefer, and Andrea Vest Ettekal. 2020. "Crafting Mosaics: PersonCentered Religious Influence and Selection in Adolescent Friendships.” Journal for the Scientific Study of Religion 59(1): 39-61.

Barber, Bonnie L., Jacquelynne S. Eccles, and Margaret R. Stone. 2001. "Whatever Happened to the Jock, the Brain, and the Princess? Young Adult Pathways Linked to Adolescent Activity Involvement and Social Identity." Journal of Adolescent Research 16(5):429-55. Bearman, Peter S., Jo Jones, and J. Richard Udry. 1997. "The National Longitudinal Study of Adolescent Health: Research Design."

Blau, Peter M. 1977. Inequality and Heterogeneity: A Primitive Theory of Social Structure. New York: Free Press.

Bojanowski, Michał and Rense Corten. 2014. "Measuring Segregation in Social Networks." Social Networks 39:14-32. 
Bolck, Annabel, Marcel Croon, and Jacques Hagenaars. 2004. "Estimating Latent Structure Models with Categorical Variables: One-step versus Three-step Estimators.” Political Analysis 12(1):3-27.

Bray, Bethany C., Stephanie T. Lanza, and Tan Xianming. 2015. Eliminating Bias in ClassifyAnalyze Approaches for Latent Class Analysis.” Structural Equation Modeling 22(1):111.

Brechwald, Whitney A. and Mitchell J Prinstein. 2011. "Beyond Homophily: A Decade of Advances in Understanding Peer Influence Processes." Journal of Research on Adolescence 21(1):166-79.

Brener, Nancy D. and Janet L. Collins. 1998. "Co-Occurrence of Health-Risk Behaviors among Adolescents in the United States." Journal of Adolescent Health 22:209-13.

Burdette, Amy M., Belinda L. Needham, Miles G. Taylor and Terrence D. Hill. 2017. "Health Lifestyles in Adolescence and Self-Rated Health into Adulthood." Journal of Health \& Social Behavior 58(4):520-36.

Cockerham, William C. 2005. "Health Lifestyle Theory and the Convergence of Agency and Structure." Journal of Health and Social Behavior 46:51-67.

Cockerham, William C. 2013. "Bourdieu and an Update of Health Lifestyle Theory." Pp. 127154 in Medical Sociology on the Move. Dordrecht: Springer.

Cohen-Cole, Ethan and Jason M. Fletcher. 2008. "Detecting Implausible Social Network Effects in Acne, Height and Headaches: Longitudinal Analysis." BMJ 337:a2533.

Daw, Jonathan, Rachel Margolis, and Laura Wright. 2017. "Emerging Adulthood, Emergent Health Lifestyles: Sociodemographic Determinants of Trajectories of Smoking, Binge 
Drinking, Obesity, and Sedentary Behavior." Journal of Health \& Social Behavior 58(2):181-97.

de la Haye, Kayla, Garry Robins, Philip Mohr, and Carlene Wilson. 2011. "How Physical Activity Shapes and Is Shaped by Adolescent Friendships." Social Science and Medicine 73(5):719-28.

de la Haye, Kayla, Harold D. Green, David R. Kennedy, Michael Pollard, and Joan S. Tucker. 2013. "Selection and Influence Mechanisms Associated with Marijuana Initiation and Use in Adolescent Friendship Networks." Journal of Research on Adolescence 23:47486.

Ennett, Susan T., Karl E. Bauman, Andrea Hussong, Robert Faris, Vangie A. Foshee, Li Cai, and Robert H.. 2006. "The Peer Context of Adolescent Substance Use: Findings from Social Network Analysis." Journal of Research on Adolescence 16(2):159-86.

Frank, Kenneth A., Chandra Muller, Kathryn S. Schiller, Catherine Riegle-Crumb, Anna Strassmann Mueller, Robert Crosnoe, and Jennifer Pearson. 2008. "The Social Dynamics of Mathematics Coursetaking in High School." American Journal of Sociology 113(6): $1645-1696$.

Franken, Aart, Mitchell J. Prinstein, Jan Kornelis Dijkstra, Christian E. G. Steglich, Zeena Harakeh, and Wilma A. M. Vollebergh. 2016. "Early Adolescent Friendship Selection Based on Externalizing Behavior: The Moderating Role of Pubertal Development. The Snare Study." Journal of Abnormal Child Psychology 44(8):1647-57.

Frech, Adrienne. 2012. "Healthy Behavior Trajectories between Adolescence and Young Adulthood." Advances in Life Course Research 17(2):59-68. 
Friedkin, Noah E. and Karen S. Cook. 1990. "Peer Group Influence." Sociological Methods \& Research 19(1):122-43.

Frohlich, Katherine L. and Louise Potvin. 1999. "Collective Lifestyles as the Target for Health Promotion." Canadian Journal of Public Health 90(S1):S11-14.

Gest, Scott D., D. Wayne Osgood, Mark E. Feinberg, Karen L. Bierman, and James Moody. 2011. "Strengthening Prevention Program Theories and Evaluations: Contributions from Social Network Analysis." Prevention Science 12(4):349-60.

Goldberg, Amir and Sarah K. Stein. 2018. "Beyond Social Contagion: Associative Diffusion and the Emergence of Cultural Variation." American Sociological Review 83(5): 897-932.

Green, Harold D., Mariana Horta, Kayla de la Haye, Joan S. Tucker, David R. Kennedy, and Michael Pollard. 2013. "Peer Influence and Selection Processes in Adolescent Smoking Behavior: A Comparative Study." Nicotine \& Tobacco Research 15(2):534-41.

Haas, Steven A. and David R. Schaefer. 2014. "With a Little Help from My Friends: Asymmetrical Social Influence on Adolescent Smoking Initiation and Cessation." Journal of Health and Social Behavior 55(2):126-43.

Huisman, Mark, and Christian Steglich. 2008. "Treatment of Non-Response in Longitudinal Network Studies.” Social Networks 30:297-308.

Jung, Tony and KAS Wickrama. 2008. "An Introduction to Latent Class Growth Analysis and Growth Mixture Modeling." Social and Personality Psychology Compass 2(1): 302-317.

Kandel, Denise B. 1978. "Homophily, Selection, and Socialization in Adolescent Friendships." American Journal of Sociology 84:427-36. 
Kirke, Deirdre M. 2004. "Chain Reactions in Adolescents' Cigarette, Alcohol and Drug Use: Similarity through Peer Influence or the Patterning of Ties in Peer Networks." Social Networks 26:3-28.

Krueger, Patrick M., Tajudaullah Bhaloo, and Pauline Vaillancourt Rosenau. 2009. "Health Lifestyles in the United States and Canada: Are We Really So Different?." Social Science Quarterly 90(5):1380-402.

Lawrence, Elizabeth M., Stefanie Mollborn and Robert A. Hummer. 2017. "Health Lifestyles across the Transition to Adulthood: Implications for Health." Social Science \& Medicine 193:23-32.

Lewis, Kevin and Jason Kaufman. 2018. “The Conversion of Cultural Tastes into Social Network Ties.” American Journal of Sociology 123(6): 1684-1742.

Liefbroer, Aart C. and Cees H. Elzinga. 2012. "Intergenerational Transmission of Behavioural Patterns: How Similar Are Parents' and Children's Demographic Trajectories?". Advances in Life Course Research 17(1):1-10.

Linzer, Drew and Jeffrey Lewis. 2011. "PoLCA: An R Package for Polytomous Variable Latent Class Analysis." Journal of Statistical Software 42(10):1-29.

Lospino, Joshua A. 2012. "Statistical Models for Social Network Dynamics." PhD, Statistics, University of Oxford.

Marsden, Peter V. 1988. "Homogeneity in Confiding Relations." Social Networks 10:57-76. McCutcheon, Allen L. 1987. Latent Class Analysis: SAGE. 
McFarland, Daniel A., James Moody, David Diehl, Jeffrey A. Smith, and Reuben J. Thomas. 2014. "Network Ecology and Adolescent Network Structure." American Sociological Review 79(6):1088-121.

McPherson, Miller, Lynn Smith-Lovin, and James M. Cook. 2001. "Birds of a Feather: Homophily in Social Networks." Annual Review of Sociology 27:415-44.

Mollborn, Stefanie, Benjamin W. Domingue, and Jason D. Boardman. 2014a. "Norms as GroupLevel Constructs: Investigating School-Level Teen Pregnancy Norms and Behaviors." Social Forces 93(1):241-67.

Mollborn, Stefanie, Laurie James-Hawkins, Elizabeth Lawrence, and Paula Fomby. 2014b. "Health Lifestyles in Early Childhood." Journal of Health and Social Behavior 55(4):386-402.

Mollborn, Stefanie, Elizabeth Lawrence, Laurie James-Hawkins, and Paula Fomby. 2014c. "How Resource Dynamics Explain Accumulating Developmental and Health Disparities for Teen Parents' Children." Demography 51(4):1199-224.

Mollborn, Stefanie, Bethany Rigles, and Jennifer A. Pace. 2020. “'Healthier Than Just Healthy': Families Transmitting Health as Cultural Capital.” Social Problems. Accessible at https://academic.oup.com/socpro/advancearticle/doi/10.1093/socpro/spaa015/5827866?searchresult=1.

Moody, James. 2001. "Race, School Integration, and Friendship Segregation in America." American Journal of Sociology 107(3):679-716.

Moody, James, Mark E. Feinberg, D. Wayne Osgood, and Scott D. Gest. 2010. "Mining the Network: Peers and Adolescent Health." Journal of Adolescent Health 47(4):324-26. 
Resnick, Michael D., Peter S. Bearman, Robert W. Blum, Karl E. Bauman, Kathleen M. Harris, Jo Jones, Joyce Tabor, Trish Beuhring, Renee E. Sieving, Marcia Shew, Marjorie Ireland, Linda H. Bearinger, and J. Richard Udry. 1997. "Protecting Adolescents from Harm: Findings from the National Longitudinal Study on Adolescent Health." Journal of the American Medical Association 278(10):832-43.

Ripley, Ruth M, Tom AB Snijders, Z. Boda, A. Voros, and Paulina Preciado. 2019. "Manual for Rsiena." University of Oxford: Department of Statistics, Nuffield College.

Schaefer, David R., Olga Kornienko, and Andrew M. Fox. 2011. "Misery Does Not Love Company: Network Selection Mechanisms and Depression Homophily." American Sociological Review 75(5):764-85.

Schaefer, David R. and jimi adams. 2017. "The Coevolution of Networks and Health: Introduction to the Special Issue of Network Science." Network Science 5(3):249-56.

Shalizi, Cosma Rohilla and Andrew C. Thomas. 2011. "Homophily and Contagion Are Generically Confounded in Observational Social Network Studies." Sociological Methods \& Research 40(2):211-39.

Smith, Kirsten P. and Nicholas A. Christakis. 2008. "Social Networks and Health." Annual Review of Sociology 34:405-29.

Snijders, Tom A. B. 2001. "The Statistical Evaluation of Social Network Dynamics." Sociological Methodology 31:361-95.

Snijders, Tom A. B. 2011. "Statistical Models for Social Networks." Annual Review of Sociology 37:129-51. 
Snijders, Tom A. B., Gerhard van de Bunt and Christian E. G. Steglich. 2010. "Introduction to Stochastic Actor-Based Models for Network Dynamics." Social Networks 32:44-60. Snijders, Tom A. B., Alessandro Lomi, and Vanina Jasmine Torló. 2013. "A Model for the Multiplex Dynamics of Two-Mode and One-Mode Networks, with an Application to Employment Preference, Friendship, and Advice." Social Networks 35(2):265-76.

Stead, Martine, Laura McDermott, Anne Marie MacKintosh, and Ashley Adamson. 2011. "Why healthy eating is bad for young people's health: Identity, belonging and food." Social Science \& Medicine 72(7): 1131-1139.

Steglich, Christian, Tom A. B. Snijders, and Michael Pearson. 2010. "Dynamic Networks and Behavior: Separating Selection from Influence." Sociological Methodology 40(1):329_ 93.

Stets, Jan E., and Peter J. Burke. 2000. "Identity theory and social identity theory." Social Psychology Quarterly 63(3): 224-237.

Strang, David and Sarah A. Soule. 1998. "Diffusion in Organizations and Social Movements: From Hybrid Corn to Poison Pills." Annual Review of Sociology 24:265-90.

Sussman, Steve, Pallay Pokhrel, Richard D. Ashmore, and B. Bradford Brown. 2007. "Adolescent Peer Group Identification and Characteristics: A Review of the Literature." Addictive Behaviors 32(8):1602-27.

Valente, Thomas W. 2010. Social Networks and Health: Models, Methods, and Applications. New York: Oxford University Press. 
Vermut, Jeroen K. and Jay Magidson. 2002. "Latent Cluster Analysis." Pp. 89-106 in Applied Latent Class Analysis, edited by J. A. Hagenaars and A. L. McCutcheon. New York: Cambridge.

Williams, Simon J. 1995. "Theorising Class, Health and Lifestyles: Can Bourdieu Help Us?". Sociology of Health and Illness 17(5):577-604.

Wimmer, Andreas and Kevin Lewis. 2010. "Beyond and Below Racial Homophily: Erg Models of a Friendship Network Documented on Facebook." American Journal of Sociology 116(2):583-642.

Zweig, Janine M., Stacey D. Phillips, and Laura Duberstein Lindberg. 2002. "Predicting Adolescent Profiles of Risk: Looking Beyond Demographics." Journal of Adolescent Health 31(4):343-53. 
Figure 1. Conceptualizing and Modeling Peer Influence and Homophilous Selection

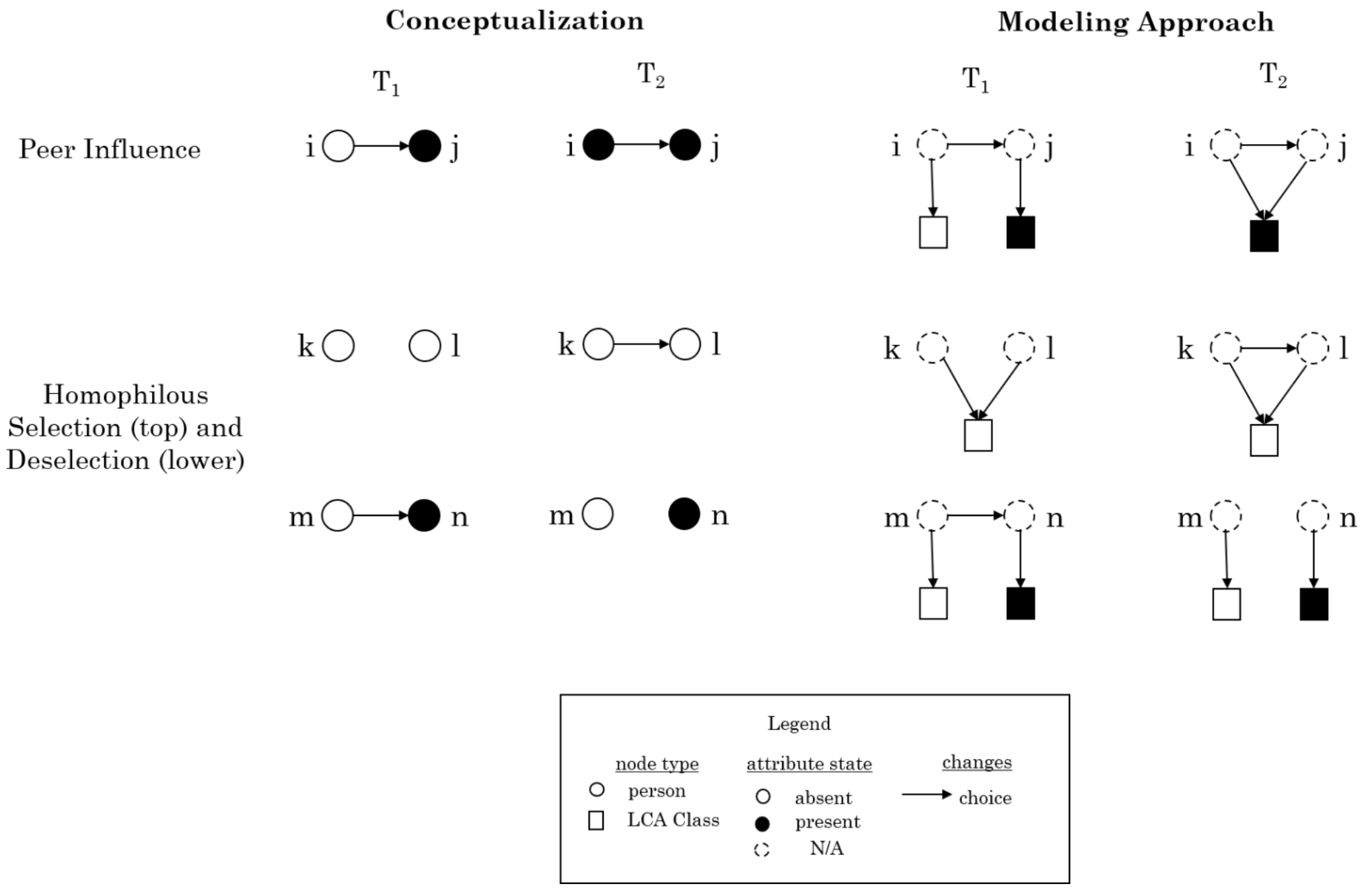




\section{Figure 2. Class-Conditional Response Probabilities from LCA, by School and Wave}
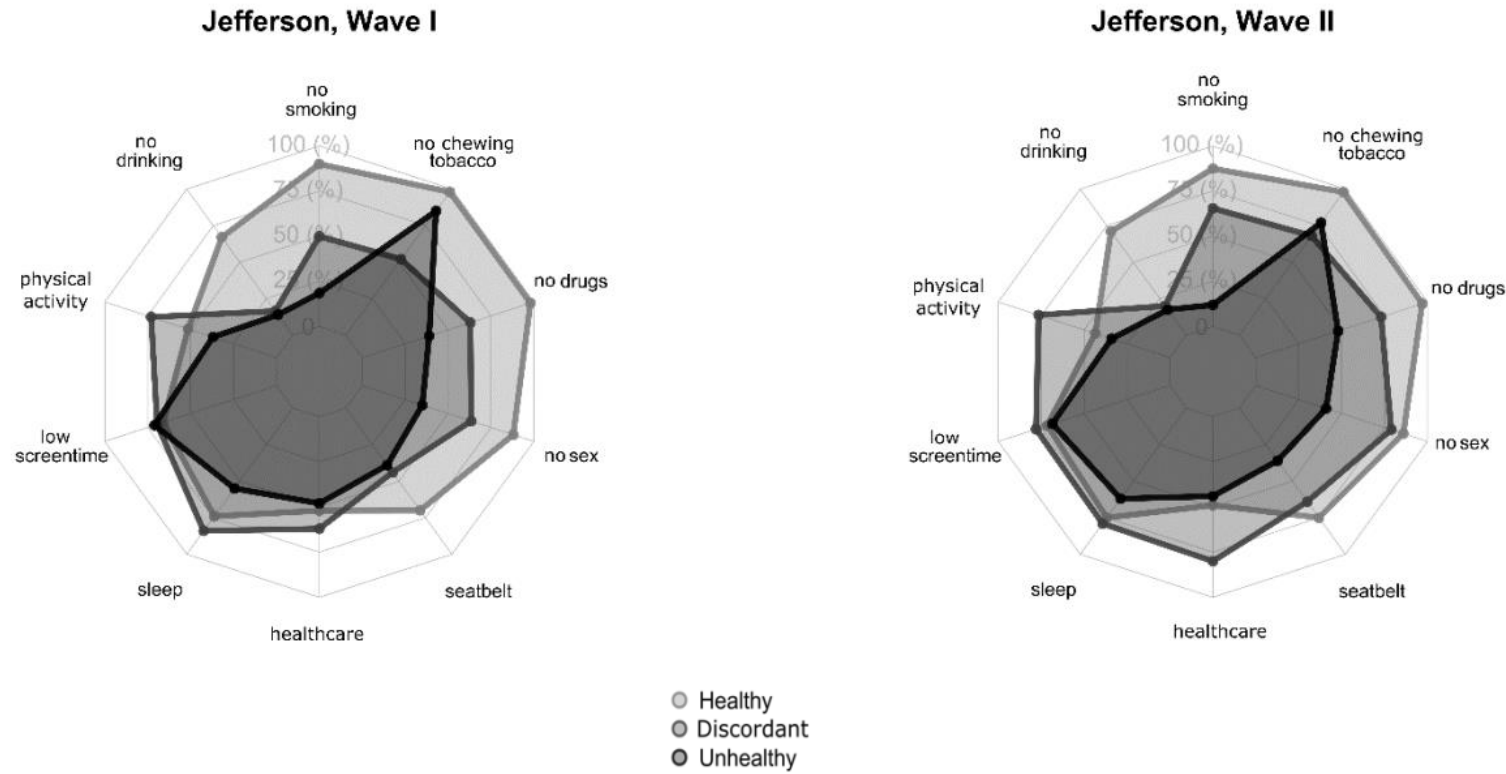

Sunshine, Wave I

Sunshine, Wave II
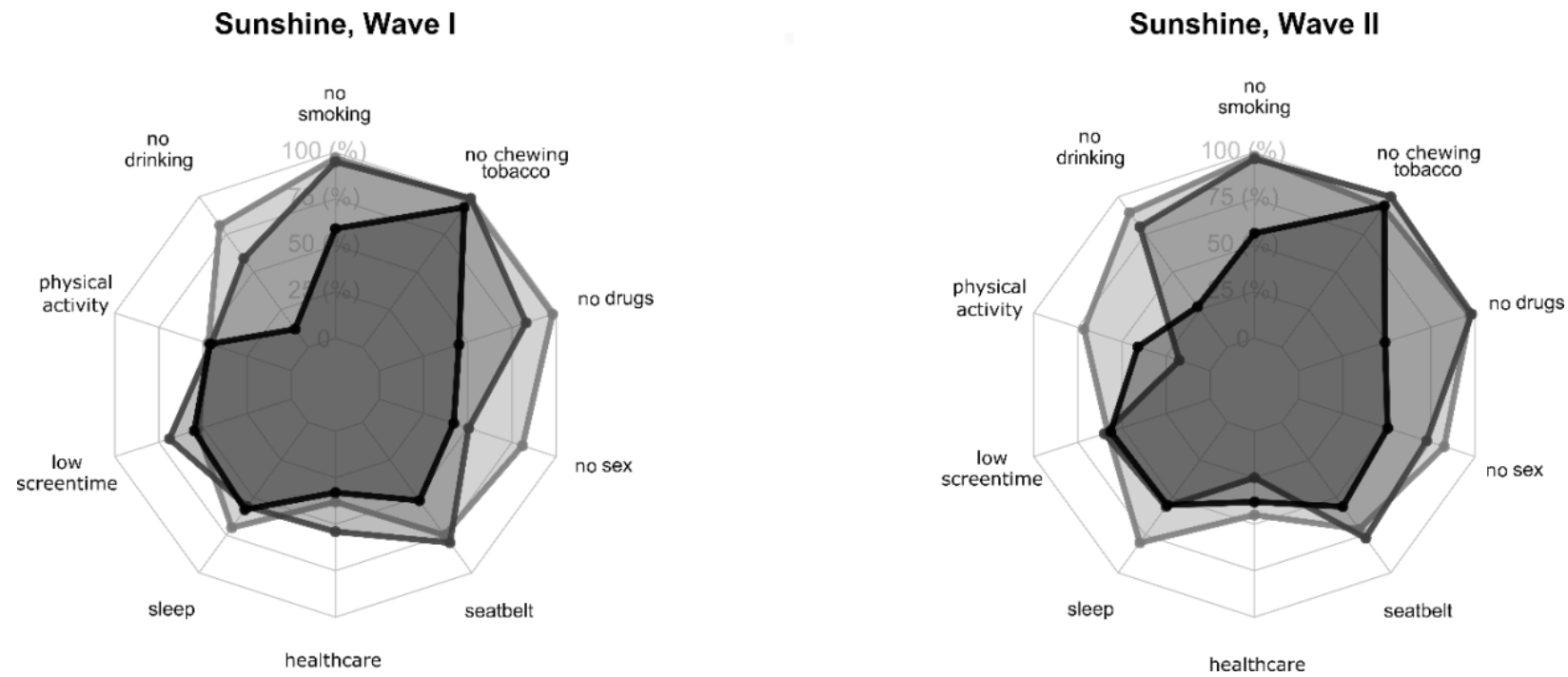

NOTES: The categorical response probabilities were re-scaled to a uniform 0-1 scale, with zero representing the lowest possible value, and 1 the highest for each variable (see details in Appendix Table A2). For trichotomous variables, this required multiplying the category-specific response probabilities by a scaling factor ( 0 for lowest category, 0.5 for moderate, and 1 for the highest). These then essentially reflect the class-conditional likelihood of selecting the highest category for each variable. For the sexual activity variable, the ordering is no sexual activity, condom at last sex, no condom at last sex. 
Figure 3. Peer Influence and Homophilous Selection of Health Lifestyles from SABM, by School

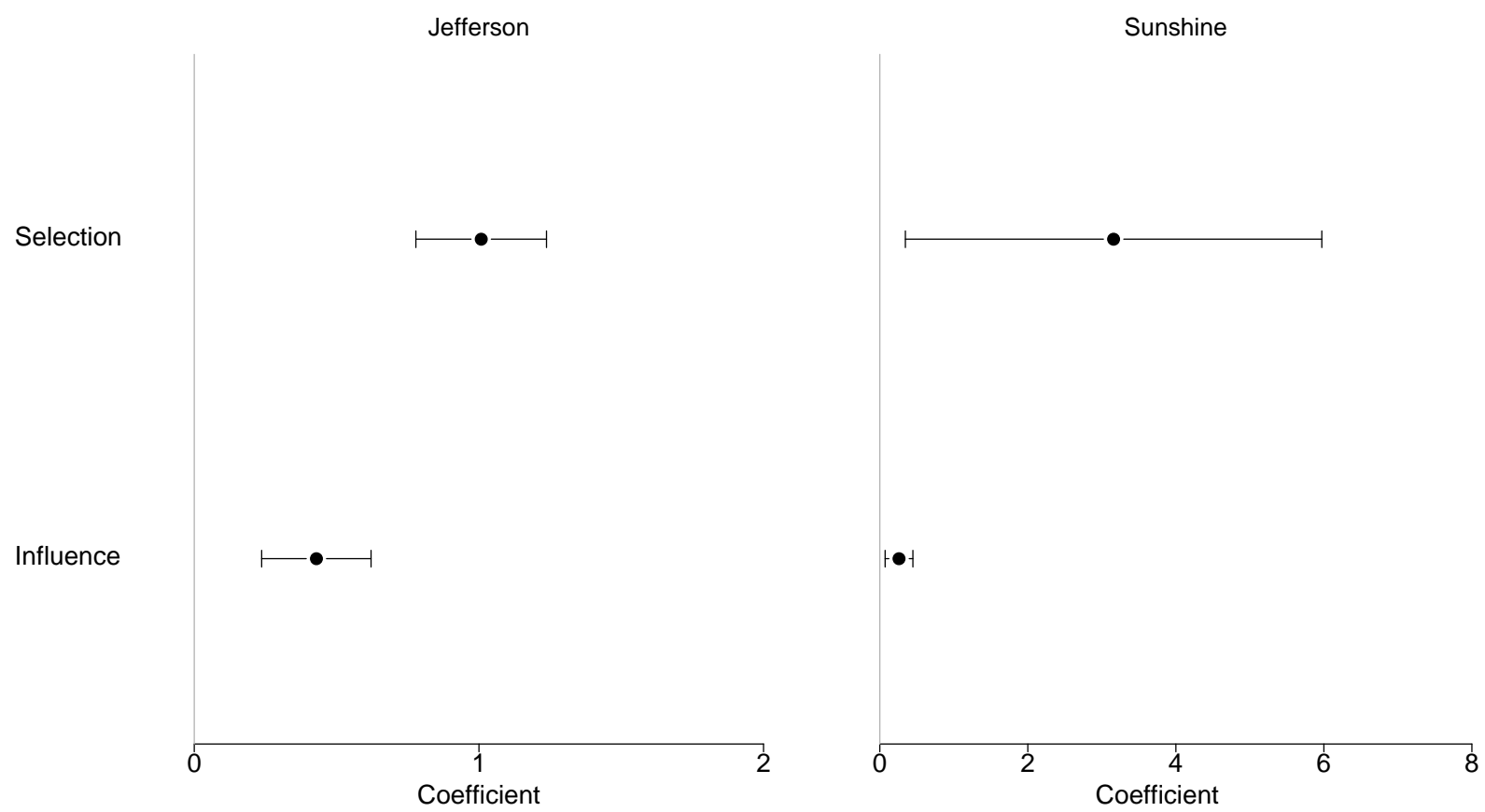

Notes: Behavior function (influence effect) controls for: age, gender, GPA, race ("Sunshine" only) \& parental education. Network (selection effect) function controls for: density, reciprocity, transitivity, (sqrt) indegree, \# of shared courses \& extracurriculars, ego/alter/similarity: gender, grade, race ("Sunshine" only) \& parental education, GPA. 
Figure 4. Health Lifestyle Assortativity Decomposition, by School

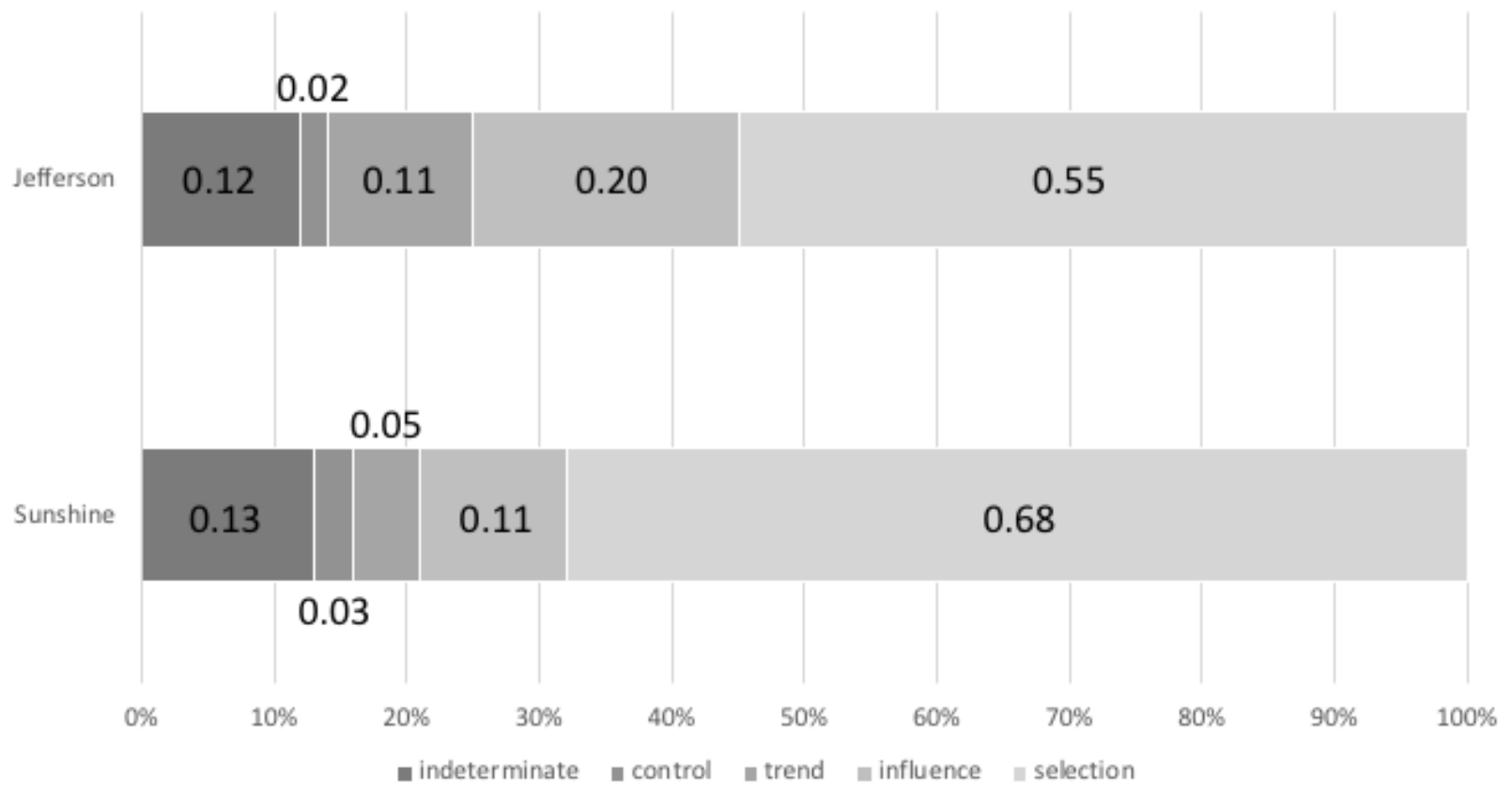

NOTES: This decomposition accounts for how much of the observed changes in health lifestyle assortativity can be attributed to four separate processes. These are (from right-to-left): homophilous selection and peer influence are the key processes of interest in the paper; the trend accounts for baseline changes in the network and LCA class membership, while control accounts for changes deriving from other factors included in the model. These four leave some proportion of the observed changes unaccounted for in the model (indeterminate). 
Table 1. Respondent Descriptive Statistics, by School and Wave

\begin{tabular}{|c|c|c|c|c|}
\hline & \multicolumn{2}{|c|}{ "Jefferson HS" } & \multicolumn{2}{|c|}{ "Sunshine HS" } \\
\hline & Wave I & Wave II & Wave I & Wave II \\
\hline \multicolumn{5}{|l|}{ Sociodemographic Characteristics } \\
\hline \multicolumn{5}{|c|}{ Age } \\
\hline $14-15$ & .27 & .06 & .10 & .01 \\
\hline $16-17$ & .49 & .59 & .62 & .55 \\
\hline $18-19$ & .23 & .35 & .28 & .44 \\
\hline Female & .47 & .48 & .48 & .50 \\
\hline \multicolumn{5}{|l|}{ Race / Ethnicity } \\
\hline White & .97 & .98 & .05 & .05 \\
\hline Black & .00 & .00 & .23 & .22 \\
\hline Hispanic & .01 & .01 & .39 & .41 \\
\hline Asian / PI & .00 & .00 & .31 & .30 \\
\hline Other & .01 & .01 & .01 & .02 \\
\hline GPA & 2.62 & 2.58 & 2.51 & 2.62 \\
\hline \multicolumn{5}{|l|}{ Parental Education } \\
\hline$<\mathrm{HS}$ & .03 & .03 & .22 & .22 \\
\hline HS Grad & .39 & .39 & .22 & .21 \\
\hline Some College & .25 & .25 & .22 & .21 \\
\hline 4y Degree & .34 & .33 & .35 & .36 \\
\hline \multicolumn{5}{|l|}{ Health Behaviors } \\
\hline \multicolumn{5}{|l|}{ Smoking } \\
\hline None & .51 & .44 & .78 & .73 \\
\hline Infrequent & .16 & .18 & .13 & .16 \\
\hline Frequent & .32 & .39 & .09 & .11 \\
\hline \multicolumn{5}{|l|}{ Drinking } \\
\hline None & .33 & .36 & .50 & .57 \\
\hline Moderate & .14 & .08 & .11 & .08 \\
\hline Heavy/Problem & .53 & .56 & .39 & .35 \\
\hline Other (chewing) Tobacco Use & .15 & .16 & .03 & .04 \\
\hline Drug Use & .28 & .27 & .21 & .21 \\
\hline \multicolumn{5}{|l|}{ Physical Activity } \\
\hline Heavy & .36 & .29 & .30 & .24 \\
\hline Moderate & .29 & .33 & .33 & .32 \\
\hline Light & .35 & .38 & .37 & .44 \\
\hline \multicolumn{5}{|l|}{ Screen Time } \\
\hline Low & .55 & .61 & .42 & .43 \\
\hline Moderate & .26 & .21 & .30 & .30 \\
\hline High & .19 & .18 & .28 & .27 \\
\hline \multicolumn{5}{|l|}{ Sexual Activity } \\
\hline None & .52 & .52 & .51 & .54 \\
\hline Condom @ last sex & .28 & .31 & .26 & .26 \\
\hline No Condom @ last sex & .20 & .17 & .23 & .20 \\
\hline Health Care Use & .52 & .53 & .39 & .35 \\
\hline Sufficient Sleep & .69 & .71 & .64 & .62 \\
\hline Regular Seatbelt Use & .55 & .57 & .68 & .67 \\
\hline $\mathbf{N}$ & 832 & 635 & 1719 & 1199 \\
\hline
\end{tabular}


Table 2. Network Descriptive Statistics, by School and Wave

\begin{tabular}{|c|c|c|c|c|}
\hline & \multicolumn{2}{|c|}{ "Jefferson HS" } & \multicolumn{2}{|c|}{ "Sunshine HS" } \\
\hline & Wave I & Wave II & Wave I & Wave II \\
\hline Out-Degree (mean) & 4.15 & 3.05 & 2.02 & 1.38 \\
\hline LCA-Class Assortativity $(\boldsymbol{\alpha})^{1}$ & 1.85 & 1.91 & 1.62 & 1.51 \\
\hline Jaccard Index ${ }^{2}$ & \multicolumn{2}{|c|}{.34} & \multicolumn{2}{|c|}{.21} \\
\hline
\end{tabular}

NOTES:

1 LCA-class based assortativity is estimated using Moody's (2001) $\alpha$ segregation index. The observed $\alpha$ 's indicate the odds of a tie existing that includes two adolescents with the same LCA class relative to the odds of a tie between two adolescents with different LCA classes. All $\alpha$ values are significant at $p<.05$.

${ }^{2}$ The Jaccard Index indicates the amount of stability observed between the two waves of network observations. Snijders et al. (2010) recommend that estimating SAB models require values greater than .20. 